\title{
Auditory priming in an implicit memory task that emphasizes surface processing
}

\author{
EDWARD F. MEEHAN and MAURA PILOTTI \\ College of Staten Island, City University of New York, Staten Island, New York
}

\begin{abstract}
Auditory priming was examined in an implicit memory task, phoneme monitoring, that emphasized surface processing. The contribution of voice to priming was investigated in single- and multiplespeaker environments by repeating studied words at test in either the same voice or different voices. Multiple-speaker environments, which preserved both acoustic and word repetition, eliminated priming when more than two voice changes between words were introduced. When voice familiarity attenuated acoustic variability, priming was reestablished in the condition in which three voices were heard. Voice changes between study and test, which eliminated acoustic repetition, also abolished priming. Word frequency affected reaction times but not priming. This demonstrated that priming entailed subword processing rather than word processing. This study demonstrates that the significance of voice in implicit memory is dependent on the level of processing required by the task and the acoustic environment.
\end{abstract}

Auditory priming occurs when a response to a repeated spoken word is facilitated without intentional recollection (Church \& Schacter, 1994). Auditory priming is therefore regarded as a behavioral correlate of implicit memory for spoken words.

Conventionally, investigations of auditory priming have relied on implicit memory procedures that require participants to report whole words rather than subword units (Church \& Schacter, 1994; Goldinger, 1992; Jackson \& Morton, 1984; Lively, 1994; Schacter \& Church, 1992). This response bias, generated by procedures such as wordstem completion, word identification, and lexical decision, renders priming dependent on the activation of whole words (Lively, 1994; Weldon, 1991) while focusing performance away from the acoustic component.

The acoustic characteristics of a speaker's voice are automatically encoded in speech perception (see Goldinger, Pisoni, \& Logan, 1991, for additional qualifications) and then become a component of the implicit memory record of spoken words in conjunction with word-level information (Goldinger, 1992). The contribution of voice information to priming, however, has been found to depend on the degree of sensitivity of the memory task to surface

\footnotetext{
Supported in part by an OMRDD Fellowship in the CSI/IBR Center for Developmental Disabilities to M. P., portions of this study were conducted in partial fulfillment of her requirements for the Ph.D. in the Department of Psychology of The City University of New York Graduate School and University Center. The authors are grateful to their students for assistance in collecting data, to Arti Itra for devising the apparatus, to the participants who kindly took part in the study, and to the speakers who volunteered their voices. The authors thank Barbara Church, Kim Kirsner, and Henry Roediger for their insightful comments, and give special acknowledgment to John Antrobus for his invaluable commentary and support. Correspondence should be addressed to either author, Department of Psychology, Sociology and Anthropology, College of Staten Island/CUNY, 2800 Victory Blvd., Staten Island, NY 10314 (e-mail: meehan-e(postbox.csi.cuny.edu, or pilotti@postbox.csi.cuny.edu).
}

processing rather than word-level processing. In fact, a voice change between a spoken word and its repetition has been shown to have a detrimental effect on auditory priming in stem completion and word identification (Church \& Schacter, 1994; Goldinger, 1992; Schacter \& Church, 1992), but not in lexical decision (Lively, 1994).

The purpose of this study is to report on an implicit memory procedure designed to eliminate the word-level bias and enhance the sensitivity to acoustic voice processing. To emphasize the subword level, the present study employed a phoneme-monitoring task at both encoding (study) and retrieval (test). Spoken words presented at study were repeated at test along with other words. Auditory priming was indexed by faster response latencies to previously studied words than to nonstudied words. A one-speaker reference condition, in which studied words were repeated at test in the same voice, was utilized to ascertain the presence of priming with this procedure.

Although it has been recognized that the detection of voice effects on auditory priming depends on the sensitivity of a memory task to surface processing, the detrimental impact of acoustic variability on the voice component of priming has not been fully appreciated. The effects of voice information on priming have been primarily obtained in multiple-speaker acoustic environments (Church \& Schacter, 1994, Experiment 1; Goldinger, 1992; Lively, 1994; Schacter \& Church, 1992), although these environments are known to impair word identification (Mullenix, Pisoni, \& Martin, 1989) and to minimize the encoding of voice (Goldinger, 1992). In the present study, it was hypothesized that, by de-emphasizing word information, as in phoneme monitoring, the sensitivity of the task to the acoustic voice component of priming would be enhanced. Consequently, the detrimental impact of acoustic variability would be accentuated. In fact, performance in a phoneme-monitoring task is disrupted by voice changes between words (Mullenix \& Pisoni, 1990). 
To assess the effect of acoustic variability on priming, voice changes between contiguous words were employed. Voice changes were produced by two, three, or four speakers. The contribution of voice characteristics to priming was measured by introducing voice changes between study and test. Lastly, it was of interest to determine if auditory priming in this task was influenced by wholeword processing. It was hypothesized that if priming is not sustained by word units, comparable priming effects should be obtained with high- and low-frequency words.

\section{METHOD}

\section{Participants}

The participants were 280 college sturdents, who had volunteered for this study. They reported English as their first language, and they had no history of speech or hearing disorder. Three additional participants, who failed to reach a $90 \%$ accuracy rate in preliminary training, were excluded from the analyses.

\section{Stimuli and Apparatus}

The stimuli consisted of 48 monosyllabic English words divided into two sets of 24 , in which 12 stimuli began with the phoneme $b$ and 12 stimuli began with $t, d$, or $p$ ( 4 of each phoneme). Each set was matched for frequency (Francis \& Kučera, 1982), such that half of the words were of high frequency (mean frequency $=226.88$, median $=151.5$ ) and half were of low frequency (mean frequency $=3.25$, median $=3.00$ ). Each set was presented as a study list equally often. The test list inciuded all 48 words, so that half were previously studied and half were not.

Each of the 48 stimuli was recorded by four speakers (two males and two females). These four speakers and two additional speakers (one male and one female) recorded 72 similar stimuli to be used for preliminary training. Lists consisted of words spoken by one, two (a male and a female), three, or four speakers.

Each stimulus was low-pass filtered at $4.8 \mathrm{kHz}$ and digitized with an $11-\mathrm{kHz}$ sampling rate by using a 16-bit analog-to-digital converter equipped with antialiasing filters, installed on an IBM-compatible 486 personal computer. A signal processing package was utilized to create a file for each word (mean length $=501 \mathrm{msec}$ ). Words were rerecorded until two naive independent observers rated them to be intelligible and clear. The presentation of the stimuli (filtered at $4.8 \mathrm{kHz}$ ) on calibrated Koss TNT/77 headphones at $75 \mathrm{~dB}$ and the recording of the participants' responses were accomplished by a custom-made program.

\section{Design}

Four same-speaker baseline conditions, consisting of one, two, three, or four speakers, were utilized. Each studied word was spoken by the same speaker during the test phase. The speakers of the preliminary training were always different from those utilized in the study and test phases. The length of the study and test lists was kept constant. Therefore, as the number of speakers increased, the total number of exposures, or familiarity, to each speaker decreased. To obtain an unconfounded measure of speaker variability, the multiple-speaker baseline conditions that demonstrated a change in priming relative to the onespeaker conditions were replaced with familiarity conditions. This was accomplished by using the speakers of the study and test phases in preliminary training and, thus, where necessary, extending training. The 72 exposures to a voice in the same one-speaker condition served as the reference point (i.e., 24 studied words and 48 test words). There were 36,24 , and 18 exposures to each speaker in the two-, three-, and fourspeaker conditions, respectively. The number of exposures to a given voice was equated to the reference by presenting in preliminary training each voice 36,48 , and 54 times, respectively.

Finally, to assess the importance of voice repetition, when priming was found in any of the above baseline or familiarity conditions, corresponding different-speaker conditions were devised. In these conditions, each study word was spoken at test in a different gender voice. Voice changes in the familiarity conditions entailed familiar voices.
In summary, the experiment comprised the following factors: priming (studied and nonstudied; within-subjects), number of speakers (one, two, three, and four speakers; between-subjects), voice (same and different; between-subjects), word frequency (high and low frequency; within-subjects), and target phoneme (presence of $b$ and absence of $b$; within-subjects)

\section{Procedure}

The experiment was presented to the participants as a study on perception, consisting of a series of brief tasks. The participants were first exposed to preliminary training, with feedback, to assure a high level of accuracy and minimize speed-accuracy tradeoffs. They were asked to listen to a list of stimuli and indicate, as quickly and accurately as possible, whether or not each stimulus began with the phoneme $b$, by pressing the right or left mouse key, respectively. An overall $90 \%$ accuracy rate was required to proceed to the study phase of the experiment.

Following exposure to the study list, a 10-min distractor task modeled after Posner, Boies, Eichelman, and Taylor's (1969) visual matching task was presented. Upon completion, the instructions regarding the phoneme-monitoring task were repeated, and the test list was presented without reference to the prior study list. No feedback was provided during either the study or the test phase.

The sequence of each trial consisted of a $500-\mathrm{msec}, 1000-\mathrm{Hz}$ tone, a 500 -msec silent interval, and the presentation of the stimulus. The order of stimulus presentation was independently randomized for each participant, and response latencies were computed from word onset. A trial ended and the next trial began after a response was made or if no response occurred after $3,000 \mathrm{msec}$.

In the one-speaker condition, words of both study and test lists were randomly presented. In all of the multiple-speaker conditions, the words were presented in a quasi-random order, such that contiguous words were never in the same voice. The speaker's voice assigned to each word was counterbalanced across participants within speaker conditions so that any word was uttered equally often by each of the four speakers. In multiple-speaker lists, an equal number of high- and low-frequency words were spoken by each voice.

\section{RESULTS}

Preliminary training maximized accuracy (overall mean accuracy $=98.65 \%$ ) and permitted response latencies to be the primary dependent variable (see Table 1). Latencies 2 standard deviations above and below each participant's mean were excluded from the analyses $(4.41 \%)$. In this study, priming was operationally defined as statistically significant faster response latencies to studied words than to nonstudied words. When priming was found in any of the experimental conditions, priming scores were analyzed. Priming scores were obtained by subtracting the mean latency of studied words from the mean latency of nonstudied words. An alpha level of .05 was applied to all post hoc LSD pairwise comparisons (Kirk, 1982).

To assess whether auditory priming occurred in each of the four same-speaker baseline conditions, a $4 \times 2$ analysis of variance (ANOVA) was conducted on response latencies, with number of speakers (one, two, three, and four) and priming (studied and nonstudied) as factors. ${ }^{1}$ Although no main effect of number of speakers was detected $[F(3,124)=0.69]$, both a main effect of priming and its interaction with number of speakers were significant $\left[F(1,124)=34.79, M S_{\mathrm{e}}=720.09, p<.0001\right.$, and $F(3,124)=4.54, M S_{\mathrm{e}}=720.09, p<.005$, respectively]. The significant interaction indicated that, in an implicit memory test emphasizing operations at the sub- 
Table 1

Mean Latency and Standard Deviation of Correct Identifications

as a Function of Priming and Speaker Condition, and Priming Scores as a Function of Speaker Condition

\begin{tabular}{|c|c|c|c|}
\hline \multirow[b]{2}{*}{ Priming } & \multicolumn{2}{|c|}{ Response Latency } & \multirow[b]{2}{*}{ Priming Score } \\
\hline & $M$ & $S D$ & \\
\hline \multicolumn{4}{|l|}{ Same One-Speaker Condition } \\
\hline Nonstudied & 714 & 121 & \\
\hline Studied & 677 & 107 & +37 \\
\hline \multicolumn{4}{|l|}{ Same Two-Speaker Condition } \\
\hline Nonstudied & 744 & 172 & \\
\hline Studied & 720 & 170 & +24 \\
\hline \multicolumn{4}{|l|}{ Same Three-Speaker Condition } \\
\hline Nonstudied & 709 & 161 & \\
\hline Studied & 696 & 146 & +13 \\
\hline \multicolumn{4}{|l|}{ Same Four-Speaker Condition } \\
\hline Nonstudied & 745 & 193 & \\
\hline Studied & 741 & 172 & +4 \\
\hline \multicolumn{4}{|c|}{ Same Three-Speaker Familiarity Condition } \\
\hline Nonstudied & 775 & 184 & \\
\hline Studied & 760 & 172 & +15 \\
\hline \multicolumn{4}{|c|}{ Same Four-Speaker Familiarity Condition } \\
\hline Nonstudied & 718 & 140 & \\
\hline Studied & 707 & 121 & +11 \\
\hline \multicolumn{4}{|c|}{ Different One-Speaker Condition } \\
\hline Nonstudied & 703 & 121 & \\
\hline Studied & 693 & 106 & +10 \\
\hline \multicolumn{4}{|c|}{ Different Two-Speaker Condition } \\
\hline Nonstudied & 704 & 131 & \\
\hline Studied & 696 & 120 & +8 \\
\hline \multicolumn{4}{|c|}{ Different Three-Speaker Familiarity Condition } \\
\hline Nonstudied & 746 & 181 & \\
\hline Studied & 757 & 180 & -11 \\
\hline
\end{tabular}

word level, priming was dependent upon the acoustic environment. Pairwise comparisons between the response latencies of nonstudied and studied stimuli at each level of the factor number of speakers showed significant priming only in the one- and two-speaker conditions. The additional operations required to process an increasingly varied and progressively less familiar acoustic environment (Mullenix \& Pisoni, 1990) disrupted the ability of voice and word repetition to sustain priming. To evaluate whether the magnitude of priming differed between one and two speakers, the priming scores of these speaker conditions were analyzed. A one-way ANOVA, with number of speakers as factor, suggested that priming was not significantly different $[F(1,62)=1.72]$.

In summary, an increment in acoustic variability coupled with diminished voice familiarity preserved priming only in the two-speaker condition. To partial out the effect of acoustic variability from the impact of decreased voice familiarity, the three- and four-speaker conditions were replaced with their corresponding familiarity conditions (see Table 1). A $4 \times 2$ ANOVA was performed on response latencies, with number of speakers (same one, two, three, and four) and priming (studied and nonstudied) as factors (see Note 1). The data showed no main effect of number of speakers $[F(3,124)=1.37]$, but did show a main effect of priming and its significant interaction with number of speakers $\left[F(1,124)=40.94, M S_{\mathrm{e}}=743.92, p<.0001\right.$, and $F(3,124)=3.01, M S_{\mathrm{e}}=743.92, p<.033$, respectively]. Pairwise comparisons between the response la- tencies of nonstudied and studied stimuli at each level of the factor number of speakers showed that priming occurred in the one- and two-speaker conditions, as indicated earlier, and in the three-speaker familiarity condition. To assess whether priming in these three conditions was a function of the number of speakers, a one-way ANOVA was conducted on priming scores. No effect of number of speakers was observed $[F(2,93)=2.77]$. Although the three- and four-speaker baseline conditions did not produce significant priming effects, when voice familiarity was equated to the one-speaker reference, the detrimental impact of acoustic variability on priming was limited to the four-speaker familiarity condition.

To investigate whether priming was sensitive to studyto-test voice changes, response latencies of the samespeaker conditions that produced significant priming effects (one speaker, two speakers, and three familiar speakers) were combined with those obtained in the corresponding different-speaker conditions. These response latencies were submitted to a $2 \times 2 \times 3$ ANOVA, with priming (studied and nonstudied), voice (same voice and different gender voice between study and test), and number of speakers (one speaker, two speakers, and three familiar speakers) as factors. There was a main effect of priming $\left[F(1,186)=23.86, M S_{\mathrm{e}}=767.60, p<.0001\right]$ and a significant interaction of priming with voice $[F(1,186)=$ $\left.17.05, M S_{\mathrm{e}}=767.60, p<.0001\right]$ and with number of speakers $\left[F(2,186)=4.87, M S_{\mathrm{e}}=767.60, p<.009\right]$ (all the other $F \mathrm{~s}<2.99$ ). The significant interaction of priming and 
voice demonstrated that priming was sensitive to study-totest voice changes. Pairwise comparisons indicated that these voice changes abolished priming and thus showed that voice information was a critical component of auditory priming.

Further analyses were conducted to determine the effects of word frequency and target phoneme on priming. A $2 \times 2 \times 2$ ANOVA, with priming (studied and nonstudied), word frequency (high and low), and target phoneme (presence and absence), was performed on the response latencies of the conditions that produced significant priming (same one-speaker, same two-speaker, and same three-speaker familiarity conditions). This analysis yielded main effects for priming $[F(1,95)=40.50$, $\left.M S_{\mathrm{e}}=3079.69, p<.0001\right]$, word frequency $[F(1,95)=$ $\left.34.96, M S_{\mathrm{e}}=3298.34, p<.0001\right]$, and target phoneme $\left[F(1,95)=73.24, M S_{\mathrm{e}}=7877.06, p<.0001\right]$, but no significant interactions $(F \mathrm{~s}<1.46)$. Response latencies of words beginning with $b$ were, on the average, $55 \mathrm{msec}$ faster than those of words beginning with another phoneme. Responses were, on the average, $25 \mathrm{msec}$ faster to high-frequency words than to low-frequency words. The absence of a significant interaction between priming and word frequency showed that the memory traces that supported auditory priming were independent of a wordlevel factor, such as frequency of occurrence in the language. Taken together, these findings indicated that, at test, stimuli were processed at the word level, but the memory traces that supported auditory priming resided at the subword level.

The analyses on response latencies were replicated on accuracy data. The only analysis that produced significant results was the $2 \times 2 \times 2$ ANOVA, with priming, word frequency, and phoneme type as factors. Accuracy was higher for words that began with $b$ than for those that $\operatorname{did}$ not $\left[F(1,95)=5.30, M S_{\mathrm{e}}=30.12, p<.024\right]$ (all the other $F \mathrm{~s}<3.24)$.

\section{DISCUSSION}

In summary, the same one-speaker condition produced faster response latencies to studied stimuli, in which voice repetition and word repetition were combined, than to nonstudied stimuli, for which only voice repetition occurred. When voice and word repetition were maintained in a multiple-speaker environment, where voice changes occurred between words, acoustic variability and reduced voice familiarity had a potentially detrimental effect on priming. Increased acoustic variability and reduced voice familiarity, as entailed by two voices, preserved priming. When acoustic variability further increased and familiarity decreased with three and four voices, priming was abolished. Apparently, when the encoding of acoustic information required the processing of different and progressively less familiar voices, voice and word repetition were not sufficient to support priming. When voice familiarity was equated to the one-speaker reference, the detrimental effect of acoustic variability was restricted to four voices. When voice repetition was removed from both the studied and the nonstudied stimuli, equivalent response latencies were obtained. At the level of processing entailed by this task, word repetition was dependent on voice repetition to produce auditory priming. This evidence is consistent with the reliance of phoneme-identification performance on acoustic voice information (Mullenix \& Pisoni, 1990) and supports the notion that, compared with word-level tasks, such as word identification, word-stem completion, and lexical decision (Church \& Schacter, 1994; Goldinger, 1992; Lively, 1994; Schacter \& Church, 1992), phoneme monitoring emphasizes surface levels of processing, such as those that operate on acoustic and subword information.

Although phoneme monitoring directed attention to subword units, the effect of word frequency on response latencies showed that speech perception operations entailed word-level information. The absence of an effect of word frequency on priming strengthened the idea that implicit memory for spoken words, at the level of processing entailed by phoneme monitoring, is based on surface processing rather than word-level processing (see Schacter \& Church, 1992, for additional qualifications).

In conclusion, the results of this study demonstrate that the significance of human voice in implicit memory is dependent on both the level of processing required by the task and the acoustic environment. Both factors should be considered in future investigations and computational modeling.

\section{REFERENCES}

Church, B. A., \& Schacter, D. L. (1994). Perceptual specificity of auditory priming: Implicit memory for voice intonation and fundamental frequency. Journal of Experimental Psychology: Learning, Memory, \& Cognition, 20, 521-533

FrANCIS, W., \& KuČERA, M. (1982). Frequency analysis of English usage: Lexicon and grammar. Boston: Houghton Mifflin.

GOLDINGER, S. D. (1992). Words and voices: Implicit and explicit memory for spoken words (Research on Speech Perception Rep. 7). Bloomington: Indiana University, Department of Psychology.

Goldinger, S. D., Pisoni, D. B., \& Logan, J. S. (1991). On the nature of talker variability effects on recall of spoken word lists. Journal of Experimental Psychology: Learning, Memory, \& Cognition, 17, 152162.

JACKSON, A., \& MORTON, J. (1984). Facilitation of auditory word recog. nition. Memory \& Cognition, 12, 568-574.

KIRK, R. E. (1982). Experimental design: Procedures for the behavioral sciences. Belmont, CA: Brooks/Cole.

LIVELY, E. (1994). Preserving the perceptual record: Retention of talkerspecific information in long-term memory (Research on Spoken Language Processing Rep. 9). Bloomington: Indiana University, Department of Psychology.

Mullenix, J. W., \& Pisoni, D. B. (1990). Stimulus variability and processing dependencies in speech perception. Perception \& Psychophysics, 47, 379-390.

MulleniX, J. W., Pisoni, D. B., \& Martin, C. S. (1989). The effects of talker variability on spoken word recognition. Journal of the Acoustical Society of America, 85, 365-378.

Posner, M. I., Boies, S. J., Eichelman, W. H., \& Taylor, R. L. (1969). Retention of visual and name codes of single letters. Journal of Experimental Psychology Monographs, 79 (1, Pt. 2), 1-16.

SCHACTER, D. L., \& CHURCH, B. A. (1992). Auditory priming: Implicit and explicit memory for words and voices. Journal of Experimental Psychology: Learning, Memory, \& Cognition, 18, 915-930.

WELDON, M. S. (1991). Mechanisms underlying priming on perceptual tests. Journal of Experimental Psychology: Learning, Memory, \& Cognition, 17, 526-541.

\section{NOTE}

1. Prior to this analysis, to determine whether the response latencies of nonstudied words in the same-speaker baseline conditions (one, two, three, and four speakers) were comparable, response latencies of nonstudied words were submitted to a one-way ANOVA, with number of speakers as factor. No significant effect was detected $(F<1)$. The same analysis was subsequently replicated on the response latencies of nonstudied words in the same one-speaker, same two-speaker, same threespeaker familiarity, and same four-speaker familiarity conditions. This analysis also showed no significant results $(F<1)$.

(Manuscript received June 20, 1995; revision accepted for publication May $6,1996$. 